\title{
Broken-Cloud Enhancement of Solar Radiation Absorption
}

\author{
R. N. Byrne \\ Science Applications International Corporation \\ San Diego, California
}

\author{
R.C.J. Somerville \\ Climate Research Division, Scripps Institution of \\ Oceanography \\ University of California, San Diego \\ La Jolla, California
}

\author{
B. Subasilar \\ School of Physical Sciences \\ Curtain University of Technology \\ Perth, WA 6001, Australia
}

\section{Abstract}

Two papers recently published in Science have shown that there is more absorption of solar radiation than estimated by current atmospheric general circulation models (GCMs) and that the discrepancy is associated with cloudy scenes. We have devised a simple model which explains this as an artifact of stochastic radiative transport.

We first give a heuristic description, unencumbered by mathematical detail. Consider a simple case with clouds distributed at random within a single level whose upper and lower boundaries are fixed. The solar zenith angle is small to moderate; this is therefore an energetically important case. Fix the average areal liquid water content of the cloud layer, and take the statistics of the cloud distribution to be homogeneous within the layer. Furthermore, assume that all the clouds in the layer have the same liquid water content, constant throughout the cloud, and that apart from their droplet content they are identical to the surrounding clear sky. Let the clouds occupy on the average a fraction $\mathrm{p}_{\text {cld }}$ of the volume of the cloudy layer, and let them have a prescribed distribution of sizes about some mean. This is not a fractal distribution, because it has a scale. Cloud shape is unimportant so long as cloud aspect ratios are not far from unity. Take the single-scattering albedo to be unity for the droplets in the clouds. All of the absorption is due to atmospheric gases, so the absorption coefficient at a point is the same for cloud and clear sky. Absorption by droplets is less than a $10 \%$ effect in the numerical stochastic radiation calculations described below, so it is reasonable to neglect it at this level of idealization.

Now, consider the effect of a variation in the scale of the size distribution of the clouds on the spatial distribution of solar radiation. There are two fixed sizes in the problem: the photon mean free path and the layer thickness. When the cloud size and distribution scale is much smaller than a photon mean free path, the effect of the clouds is as if the droplets that compose them were uniformly distributed. Neither cloud fraction nor cloud size matters, and the problem reduces to a plane-parallel one. Scattering is maximized, because the self-shielding is minimized; the photon path length is also maximized. Absorption is maximized because it is monotonically related to path length.

As the scale size becomes larger, inhomogeneities occur in the scene, and the top of the atmosphere albedo drops. Eventually thin spots and holes develop in the cloud layer; transmission through it increases as a result. But absorption does not respond so rapidly with increased cloud size as does transmission. Bright clouds are seen from the ground in almost all of the $2 p$ solid angle regardless of the observer's exact ground location, an indication that the cloud and below-cloud volume is still full of photon tracks. Imagine a clear column cut into the clouds, such that sunlight can stream through. The additional light that reaches the ground through this column does not have the increase in path length that results from multiple scattering, but absorption in the column is still larger than calculated by a clear-sky model. This is because the column is full of laterally-traveling photons which have emerged from the surrounding clouds. An increase in the scale of the size distribution causes an increase in intermittency of occurrence (brokenness) of cloud in the fixed thickness layer assumed here. Thus absorption, which is dependent on path length, remains near its (maximum) small-cloud value as cloud size increases, even as transmission of the solar beam increases.

Very large clouds become so localized that they lose effectiveness as light sources for each other (and for ground observers). A fraction $\left(1-\mathrm{p}_{\text {cld }}\right)$ of observers would see no 
clouds, while a fraction $\mathrm{p}_{\text {cld }}$ would be under a cloud extending to their horizons. This is the situation accurately described by the fractional cloud cover (FCC) model, a weighted combination of two plane-parallel calculations, one clear and one overcast. This arrangement of liquid water minimizes the effect of scattering, because many of the droplets are hidden deep in the interior of the large cloud, and thus shielded from the solar radiation. The incloud scattering (and increase in path length) that takes place is confined to the surface of the cloud. The cloudcloud scattering is small, because the solid angle subtended by other clouds is small, vanishing in the limit of very large but very rare clouds. The absorption thus falls as cloud size increases. Once the cloud-cloud scattering becomes negligible, FCC describes the situation adequately; there are no further cloud size effects.

Thus, in this simplistic model the absorption and albedo vary from maximum to minimum, but the absorption varies more slowly as the cloud scale size increases.

Plane parallel theory holds for small cloud size scales, and FCC holds for large ones. For intermediate cloud sizes, absorption is anomalously enhanced relative to the predictions of either of those models, but is captured by a stochastic model.
Anomalous absorption is manifested largely in the near infra-red part of the solar spectrum because, although the relative increase in path length occurs for all wavelengths, there is very little absorption in the visible solar spectrum. Absorption responds more slowly than does albedo as inhomogeneities appear in a cloud layer. This is predicted by the simple model given above and confirmed by numerical calculations. We give a translation of the result into the observational quantities of Cess et al. (1995) and Ramanathan et al. (1995).

Figure 1 plots the coefficient $\beta$ derived from our calculations against cloud fraction and cloud size. It shows the existence of the decrease implied by our model. The values quoted by Cess et al. (1995) for $\beta$ are about 0.8 for the GCMs and about 0.6 for the observations. We find values below 0.6 for the case of small dense widely scattered clouds (the region of low cloud volume fraction and $1 \mathrm{~km}$ sizes). We find $\beta=0.7$ for small or large cloud sizes, rather than the expected 0.8 , which may be due to the parameters we used in the example. The net result is that the change in $\beta$ that we find, due to stochastic effects (about 0.1) is half that reported by Cess et al. (1995).
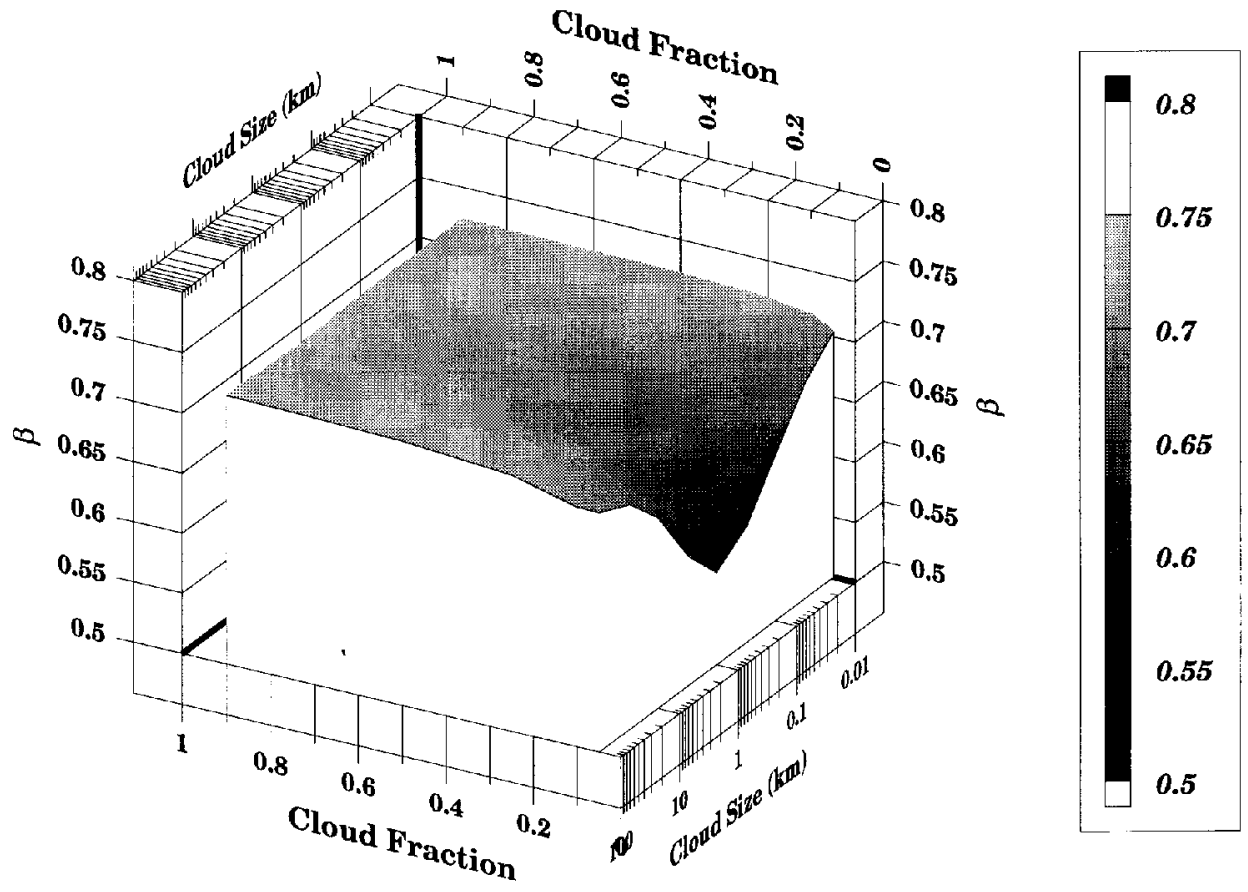

Figure 1. Cess coefficient, $\beta$, for a range of cloud sizes and fractional coverage. 
The increase in $\mathrm{f}_{\mathrm{s}}$, the measure used by Ramanathan et al. (1995), is shown in Figure 2. This figure summarizes the results of many computer runs. The same conditions that minimize $\beta$ produce values of $\mathrm{f}_{\mathrm{s}}$ greater than 1.3 , as opposed to the 1.1 we find when we neglect broken cloud effects by taking the clouds to be very small or very large. Again, our calculation produces about half the reported effect.

The numerical example presented here does not change $\beta$ or $f_{s}$ to the extent reported, but it does illustrate the existence of a one-sided bias toward smaller $\beta$ (larger $f_{s}$ ) and accounts for a significant part of the discrepancy between observations and conventional models. To ascertain the magnitude of this contribution, more research needs to be done using observed profiles and taking longterm averages.

This paper has described what we claim to be a general trend tending to support the anomalous absorption observations, but has provided numerical results for only one atmospheric profile, only one solar angle, only one cloud liquid water loading, only one surface albedo, and (for the most part) only one cloud layer at only one height and thickness. Future work should explore the variation of the effect with these parameters and provide a weighted estimate of the net result, allowing a direct comparison with the observations.

\section{Acknowledgments}

This work was supported in part by the Atmospheric Radiation Measurement (ARM) Program of the U.S. Department of Energy (DOE) under Grant No. DE-FG0292ER61638 and in part by the National Science Foundation (NSF) under Grant No. ATM 9011259. The STOCA program was developed by Somerville and Malvagi and supported in part under the ARM Program, Grant No. DE-FG03-90ER61061; the National Aeronautics and Space Administration, Grant No. NAG5-2238; the National Oceanic and Atmospheric Administration, Grant No. NA36GP0372; the NSF Grant No. ATM9114109; and a grant from the University of California Institutional Collaborative Research Program.
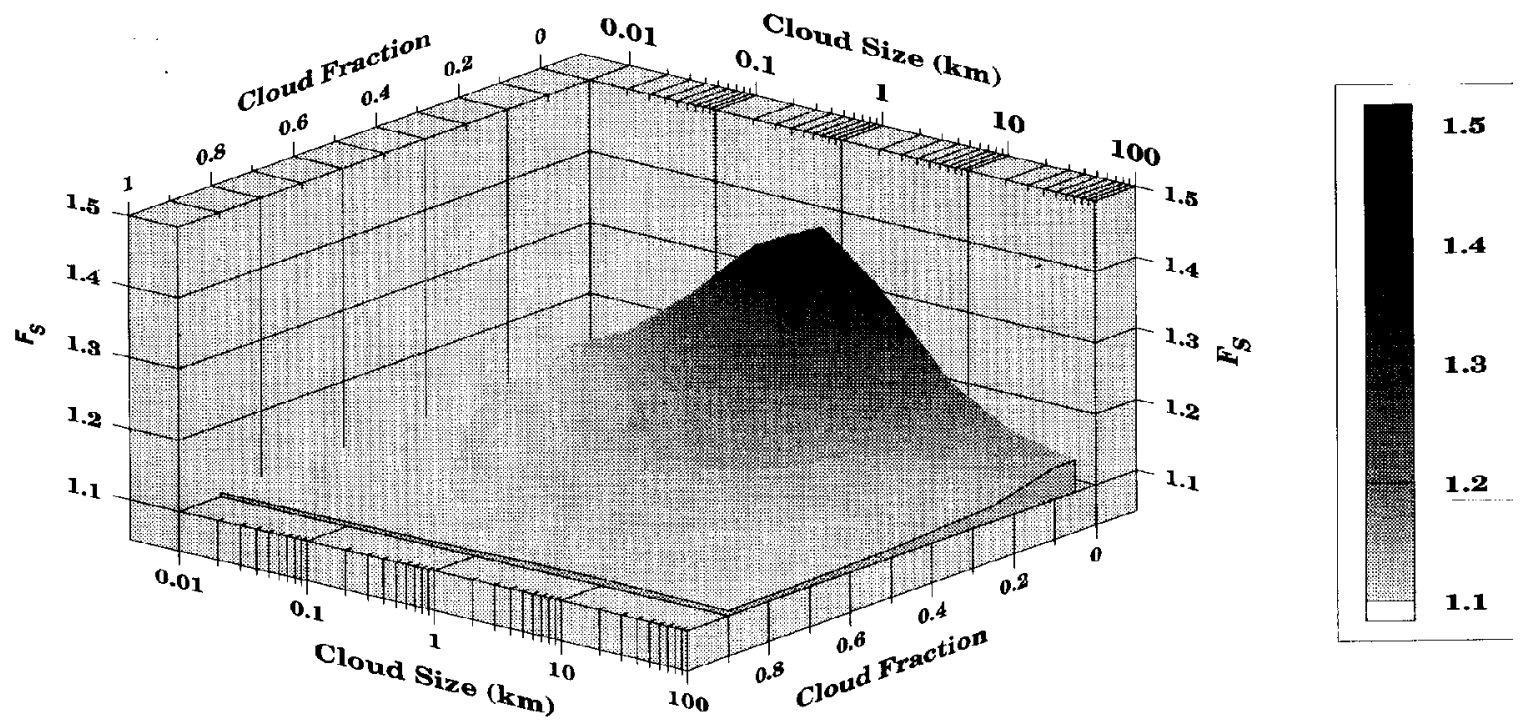

Figure 2. Ramanathan coefficient, $f_{s}$, for a range of cloud sizes and fractional coverage. 


\section{References}

Cess, R. D., M. Zhang, P. Minnis, L. Corsetti, E. G. Dutton, B. W. Forgan, D. P. Garber, W. L. Gates, J. J. Hack, E. F. Harrison, X. Jing, J. T. Kiehl, C. N. Long, J.-J. Morcrette, G. L. Potter, V. Ramanathan, B. Subasilar, C. H. Whitlock, D. F. Young, and Y. Zhou. 1995. Absorption of solar radiation by clouds: Observations versus models, Science, 267, 496-499.
Ramanathan, V., B. Subasilar, G. J. Zhang, W. Conant, R. D. Cess, J. T. Kiehl, H. Grassl, and L. Shi. 1995. Warm pool heat budget and shortwave cloud forcing: A missing physics? Science, 267, 499-503. 